\title{
Analysis of the atmospheric effect on the TRAGALDABAS high resolution Cosmic Ray detector
}

\author{
I. Riádigos ${ }^{12}$ for the Tragaldabas Collaboration * \\ ${ }^{1}$ Non-Linear Physics Group, University of Santiago de Compostela, Galicia, Spain \\ ${ }^{2}$ Instituto Galego de Física de Altas Enerxías, University of Santiago de Compostela, Galicia, \\ Spain \\ E-mail: irma.riadigos@usc.es
}

\begin{abstract}
Using ground based detectors, the accuracy in the estimation of the properties (mass, energy, direction) of high energy primary cosmic rays arriving on Earth is limited due to the effect of the atmosphere (mainly, pressure and temperature) on the measured "secondary cosmic rays". Whereas the ground pressure is quite easy to measure (a first order linear correction can be readily perform), the correction of the temperature effect on muon detectors is particularly complicated; as a matter of fact no standard method exists.

Trasgo detectors are high resolution tracking devices, providing: (i) high resolution on position and time, (ii) the capability of measuring high-multiplicity events, and (iii) some muon-electron separation. As different event topologies do correspond -usually- to different evolution of showers, then by keeping memory of the different pressure layers of the atmosphere, the analysis of the trends of different multiplicity and composition events may provide information in real time of the changes of the temperature in high levels of the atmosphere. This feature opens the door to the development of auto-calibrated detectors, able to perform a real time estimation of the temperature profile of the atmosphere, as well as a real time correction of the data by the atmospheric effect.

Some preliminary results on the correlations between cosmic rays and temperature profile of the atmosphere (using data taken with the TRAGALDABAS detector at the Univ. of Santiago de Compostela) will be shown and discussed.
\end{abstract}

36th International Cosmic Ray Conference -ICRC2019-

July 24th - August 1st, 2019

Madison, WI, U.S.A.

\footnotetext{
* for collaboration list see PoS(ICRC2019)1177
} 


\section{Introduction}

The use of muon detectors is becoming increasingly important in the solar physics research due to their versatility, accuracy and low cost compared to other big arrays of cosmic ray detectors. A good example is the Global Muon Detector Network (GMDN), which is composed of several ground-based detectors strategically located around the globe. The network represents an important tool for forecasting geomagnetic storms and it is also being used for several space weather applications. However, the use of such detectors for the research of cosmic rays variations has some limitations due to the corrections of the atmospheric effects in the secondary component of cascades.

Here, those limitations are turn into an advantage for the alternative study of the properties of the atmosphere. For such purpose, a new high performance tracking detector of small area, TRAGALDABAS (TRAsGo for the AnaLysis of the nuclear matter Decay, the Atmosphere, the earth B-field And the Solar activity), is operating at Santiago de Compostela University in Spain to study in detail the variations of hard and soft component of cosmic rays showers.

The analysis of the atmospheric variations involve mainly the barometric effect and the temperature effect, the latter being a major challenge that makes it difficult to reach a consensus on which method of correction to use. Several approachs have been proposed: the method of effective level of generation [1], the method of effective temperature [2], the integral method [3][4]. Some of them have been analyzed to find the one that best reproduces the variations observed [5] [6], concluding that the integral method tipically shows the best results.
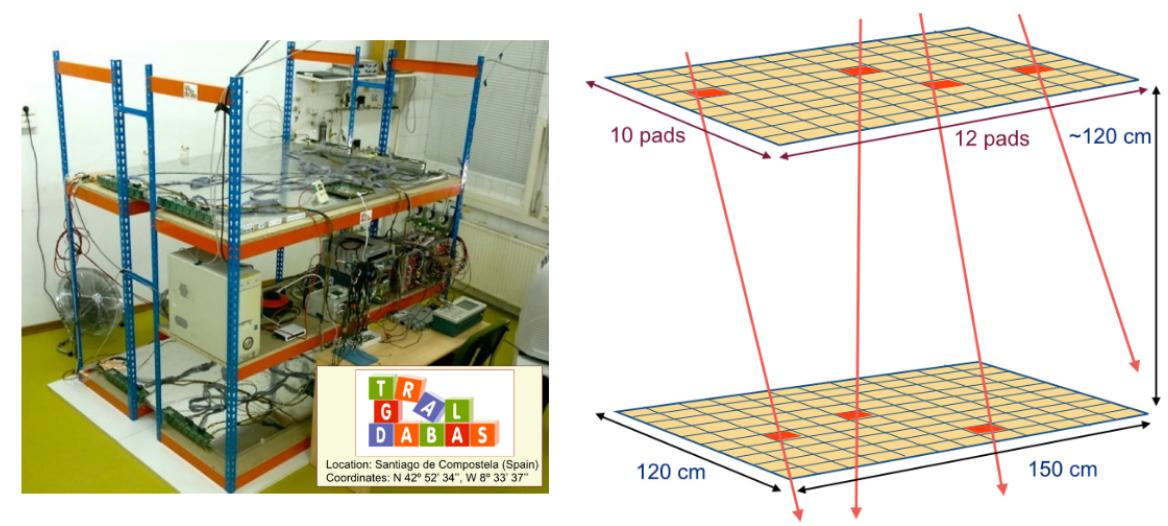

Figure 1: Lef: Picture of Tragaldabas detector in the laboratory. Right: RPC-planes scheme of the device.

\subsection{The Atmospheric Effect}

The barometric and temperature effect are the two main causes of surface variations in the intensity of cosmic rays. Usually, the barometric effect is easily computed using a single parameter, the barometric coefficient, which describes the anticorrelation between the surface pressure and cosmic ray flux variations. Moreover, this effect is divided in two different ones: negative and positive temperature effect. The negative effect is related to the muons decay when their propagation path is increased due to the expansion of the troposphere when it gets warmer in summer. So the 
observed intensity will have an opposite variation to the surface temperature. The positive effect corresponds to the atmospheric density decrease at the muon production height. A temperature increase will reduce the meson interaction probability so they will tend to decay giving rise to a muons increase. The relevance of each effect in the different layers of the atmosphere depends on the energy of the measured muons. The positive effect dominates when high energy muons are measured (underground detectors) because they are able to transverse the atmosphere without decaying due to the fact that the decay probability is proportional to their energy. When treating with low energy muons (grond-based detetors), they have a higher probability to decay so they will be dominated by the negative effect.

The contribution of all these processes is described by the temperature coefficients [4][7][8] which provide the response of the intensity variation at the level of detection when the temperature at a certain height has changed. These coefficients indicate that for low energy muons the negative effect dominates through the whole atmosphere.

Over the years, an attempt to correlate the intensity variations of cosmic rays with the different atmospheric layers has been done. However, when the results are compared between different stations of measurement having similar detetors configuration, there are several discrepancies [6].

When the correlation between the cosmic rays variations and the temperature variations at a certain atmospheric level is calculated $\left(\frac{\Delta N}{N}=\alpha_{p} \frac{\Delta T_{p}}{T_{p}}\right)$, the regression coefficients can not be directly compared between detectors placed at different locations. The reason is that the atmospheric configuration differs from one place to another.

For example, the cooler air forms a more compact atmosphere in polar regions. Then, while the tropopause is located at $17 \mathrm{~km}$ in the equator, it is lower in the poles, around $9 \mathrm{~km}$. In terms of pressure levels, $225 \mathrm{hPa}$ is the level taken as a global standard reference for the tropopause. Nonetheless, this can be misleading because the tropopause may be in any position between 100 and $400 \mathrm{hPa}$ during the year.

In the South Pole, it has a seasonal variation between 225 and $320 \mathrm{hPa}$, with a mean value of $270 \mathrm{hPa}$. That is, it is slightly lower than the tropopause in Santiago de Compostela, for instance.

From this point of view, comparisons should not be made between the values $\alpha_{p}$ of different detectors for the same pressure level. That is why in most cases, it is convenient the use of an effective coefficient $\alpha_{e f f}$ for such purpose. This coefficient provides a net value of the total atmospheric effect, overcoming the obstacles of both the difference between the pressure levels and the different correlations between the layers of the atmosphere unique for each geographic location (different atmospheric configurations).

On the other side, when the structure of the seasonal variations is analysed, it has already been reported a seasonal antiphase between the ground temperature and the temperature close to the altitude of maximun production of secondary cosmic rays $(\sim 100 \mathrm{hPa})$. This scenario, however, is not true for the entire globe. For the IceCube experiment location (South Pole), the sesasonal variation of temperature at $100 \mathrm{hPa}$ is in phase with the other atmospheric layers. This condition is found to be true for high latitudes $\left(>40^{\circ}\right)$.

Therefore, this situation combined with the temperature coefficients as a function of altitude results in a different magnitude of the total atmospheric effect for each detector.

Our goal is to analyze the possibility that a detector with capability of separating multiplicites and distinguish muons from electrons (using cascades properties) can provide information of dif- 
ferent layers of the atmosphere.

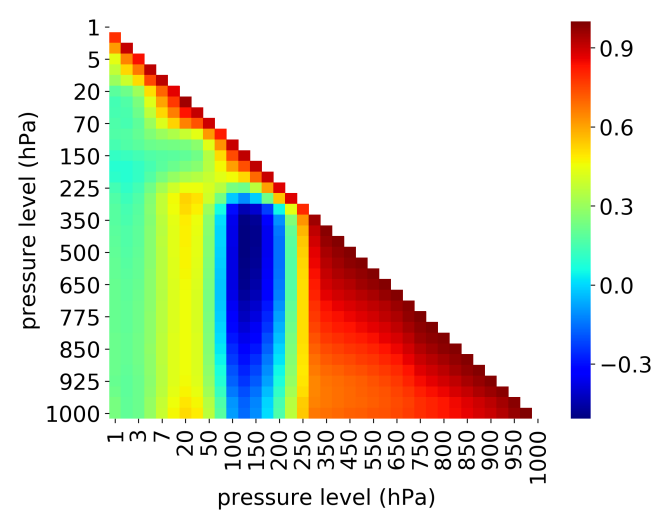

Figure 2: Correlation matrix of the temperature variations of the isobaric levels for the period between 1985-2015.

\section{Materials and Methods}

\subsection{The TRAGALDABAS detector}

A medium-size tRPC detector with an active area of $1.5 \times 1.2 \mathrm{~m}^{2}$ of high resolution $\left(\sigma_{x, y} \sim\right.$ 3, $\sigma_{t} \sim 300 \mathrm{ps}, \sigma_{\theta} \sim 2.5^{\circ}$ ), called TRAGALDABAS, was installed on the first floor of a twofloor building of the Physics Department of the University of Santiago de Compostela ( $260 \mathrm{~m}$ above sea level, $42^{\circ} 52^{\prime} \mathrm{N} 8^{\circ} 33^{\prime} \mathrm{W}$ ). Timing resistive plate chamgers (or tRPCs) are prime detectors when aimed at large area coverage with ultimate time-of-flight resolution. Due to its granularity and versatile trigger, this device can select low and high multiplicity events belonging to charged particles, by individually identifying and reconstructing each of its constituent tracks.

The detector can be labeled as a small size detector due to its very small size, near to $2 \mathrm{~m}^{2}$, unlike other detectors. For example, MuSTAnG has a surface area of $4 \mathrm{~m}^{2}$ and another one located at Mount Norikura in Japan, which is based on proportional tubes, counts with a surface area of 20 $m^{2}$.

The TRAGALDABAS has 4 RPC planes placed horizontally at different distances. It is running since 2015 with a room temperature which is kept stable around $20 \pm 0.5^{\circ} \mathrm{C}$.

A powerful tool for electron/muon track identification called MIDAS is being developed [9], even it is not implemented yet. However, it has been estimated that the detector has a muon energy threshold of $\sim 120 \mathrm{MeV}$ and $\sim 200 \mathrm{MeV}$ for electrons.

\subsection{Temperature Data}

The vertical temperature profiles for Santiago de Compostela were obtained from the European Centre for Medium-Range Weather Forecast reanalysis, ERA-INTERIM [10], at 37 isobaric levels $(1000,975,950,925,900,875,850,825,800,775,750,700,650,600,550,500,450,400,350$, $300,250,225,200,175,150,125,100,70,50,30,20,10,7,5,3,2,1 \mathrm{hPa})$. with a horizontal spatial resolution of $0.125^{\circ}$ and a temporal resolution of $6 \mathrm{~h}$. 


\subsection{Cosmic Ray Data}

Two planes, one on top of each other at a distance of $\sim 120 \mathrm{~cm}$, were used in the first period of the detector commisioning. Secondary particles are measured making coincidences of at least one signal in the upper and in the lower plane when having a velocity compatible with the speed of light (Fig. 1). Then, they are grouped by multiplicities $M$ depending on the amount of hits per event: M1 for one single hit in the upper and lower plane, two hits are classified as M2, and so on.

The data sample used for this analysis goes from autumn of 2015 until winter of 2016, with several device maintenance shutdowns in between.

\subsection{Principal Component Analysis of the Atmosphere}

As already mentioned, temperature coefficients have been calculated with high accuracy for different energy thresholds, angles of arrival, parameters, and so forth. This method always is adapted to the detector itself. However, these coefficients are sometimes difficult to estimate for a specific device and they have to be experimentally obtained in this case.

The temperature variations of the different layers of the atmosphere are strongly correlated as can be seen in Fig. 2. In general, the tropopause variations behaves opposite to the tropopause and stratosphere (antiphase). Then, the ordinary linear regression analysis is difficult to apply due to this multicollinearity. Approaches like the method of effective level of generation proposed by Duperier consists in a linear regression equation depending on the barometric pressure, the height at $100 \mathrm{hPa}$ and the ground temperature, might lead to low accuracy regression coefficients if multicollinearity is present. These coefficients could depend on the mean atmospheric properties of the observation period and their values would change from one season to another.

The principal component analysis (PCA) can be used to study the relations between the different layers of the atmosphere because is able to convert a set of correlated variables into a set of uncorrealted variables called principal components (PC). This method allows to study the behavior of the atmospheric temperature variations for Santiago de Compostela.

A training dataset of the temperature profiles in Santiago from a time series of the last 30 years is used to calculate de PCs of the temperature variations. The choice of this long period helps to reduce the effect of the variability yeart-to-year of the data, specially when exceptional events take place during the period of analysis.
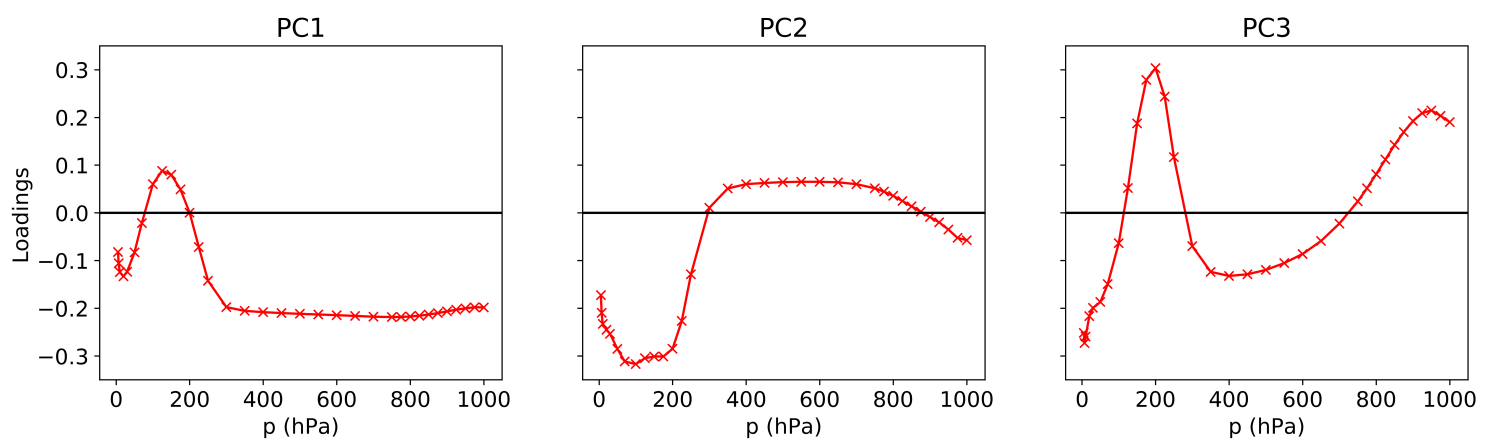

Figure 3: Factor loadings for the three principal components with largest variance. 
Fig. 3 shows the factor loadings for the first three principal components. The first component represents the seasonal changes of the temperature, reflecting the general correlations between the different layers, as previously shown in Fig. 5. Therefore, the first component is proportional to the average behavior of the atmosphere, being the temperatures in the troposphere (300-1000 hPa) the most important, i.e., equal weights. The second component is dominated by the variations of the stratosphere, representing the large temperature warming and coolings of the winter periods, such as Stratospheric Warmings. The third one is a mixture of both behaviours. These three components explain the $\sim 85 \%$ of total variance (according to general PCA criteria, the chosen components should explain between $70-90 \%$ of the total variance). Components with smaller variance have behaviours with difficult interpretation.

Owing to this, the following isobaric levels are chosen for the study of atmospheric variations in the next section: $950,700,150,30$ and $2 \mathrm{hPa}$.

\section{Data Analysis}

We proceed to analyse the different correlations between the atmospheric variations of temperatures and heights for multiplicity rates $\mathrm{M} 1, \mathrm{M} 2, \mathrm{M} 3$ and $\mathrm{M} \geq 3$. Their relative variations of rates has been standarized $\left(M_{i}-\bar{M}_{i}\right) / \sigma\left(M_{i}\right)$ and fractions between different multiplicities are also analysed.

Data is divided into 4 periods due to different detector setups during the adquisition.

\section{Barometric effect}

Before, barometric effect has to be removed. In first order approximation, the data is corrected following a linear regression between relative rate change $\Delta M_{i} / \bar{M}_{i}$ and the surface pressure $\Delta P$

$$
\frac{\Delta M_{i}}{\bar{M}_{i}}=\beta \cdot \Delta P
$$

where $\beta$ is the barometric coefficient. The values of the coefficients are shown in Table 1 . The values show a minimum for M1, with an increase in M2 and it decreases again for M3. This drop in the value of M3 could mean that this multiplicity is main composed by electrons, which are less affected by the barometric pressure.

\begin{tabular}{c|c|c|c|c} 
& M1 & M2 & M3 & Mn \\
\hline$\beta(\% / \mathrm{hPa})$ & -0.55 & -1.4 & -0.9 & -1.2
\end{tabular}

Table 1: Baromteric coefficients for the multiplicities

Fig. 4 shows the different periods corrected by pressure. It seems that multiplicities show similar trends in the variations. Besides, statistics decrease showing more fluctuations at higher multiplicities. The similarity in the tendencies may be indicating that the multiplicites are produced by primaries with energy ranges that are very close. From now, we will only show results of M1. 

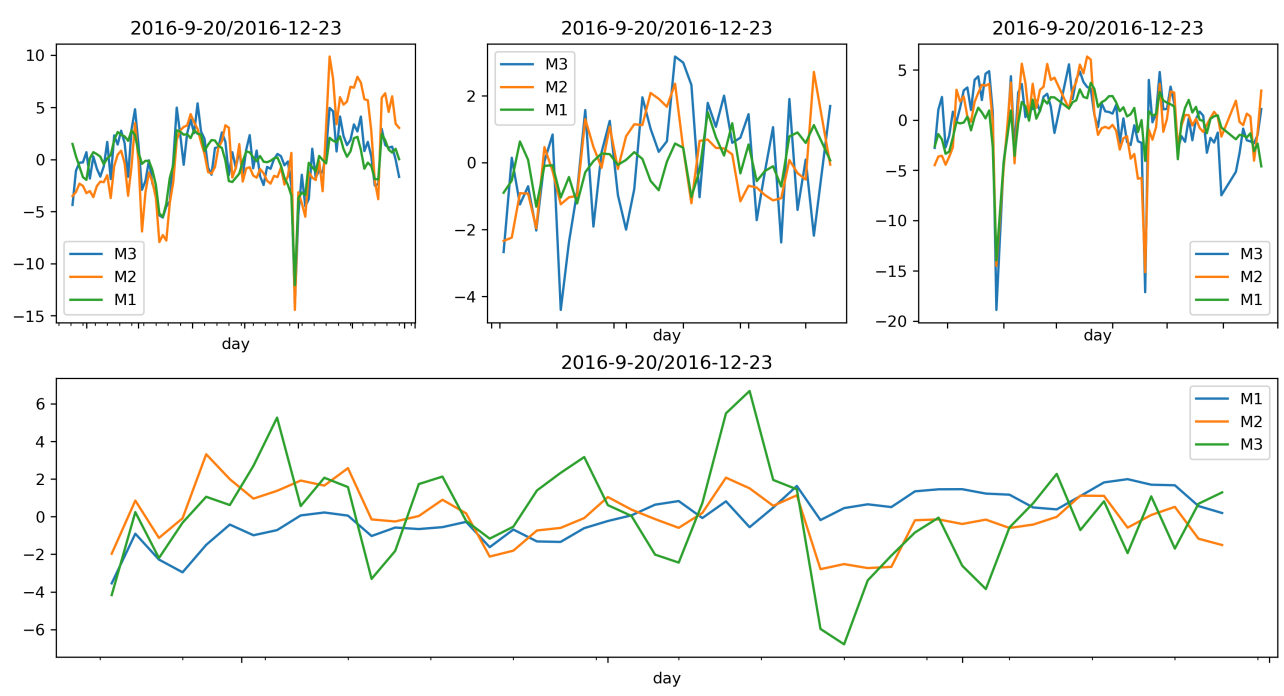

Figure 4: Different data periods showing the relative variation of multiplicity rates (M1,M2,M3) corrected by pressure.

\section{Atmospheric effect}

Correlations between atmospheric parameters (temperature and height) for the selected isobaric levels and M1 rates are studied for the different periods. No significant correlation is observed for any of the parameters.

\section{Conclusions}

Only slightly differences are observed between multiplicities. On the one hand, the similarity of energy ranges between multiplicites might be the cause and then, higher multiplicities would be needed to observed any difference. On the other hand, this could mean that the accuracy of data correction should be improved, such as efficiency and acceptance corrections, or the use of more than two planes.

Since no significant correlations have been observed with the atmospheric parameters, an improvement in the correction method should be done. Other possibility is the influence of the electronic component. Since the variations in the soft component are the opposite of muon variations, if a notable abundance of electrons with respect to muons is present, the total negative effect of the hard component would be compensated with the positive effect of soft component. Consequently, the detector would barely need the temperature corrections.

\section{References}

[1] A. Duperier, The meson intensity at the surface of the Earth and the temperature at the production level, Proc. Phys. Soc 62A 684-696 (1949).

[2] P. Barret et al, Interpretation of cosmic-ray measurements for underground, Rev. Mod. Phys 24133 (1952). 


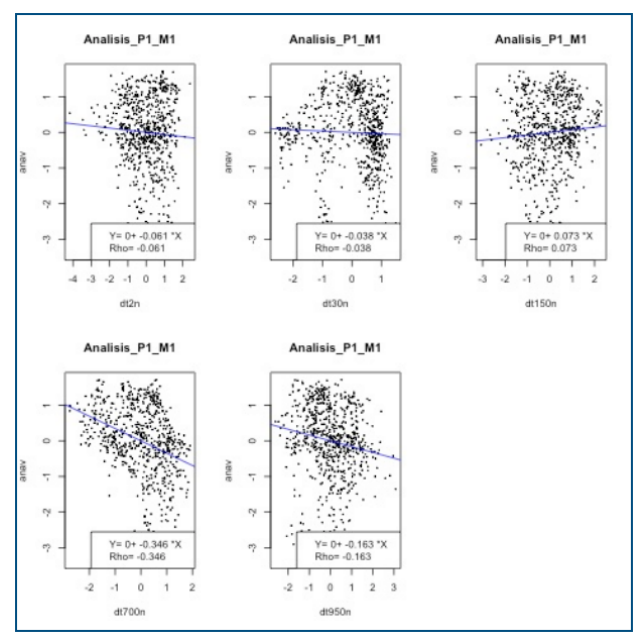

(a) Period 1a correlations with $\mathrm{T}$

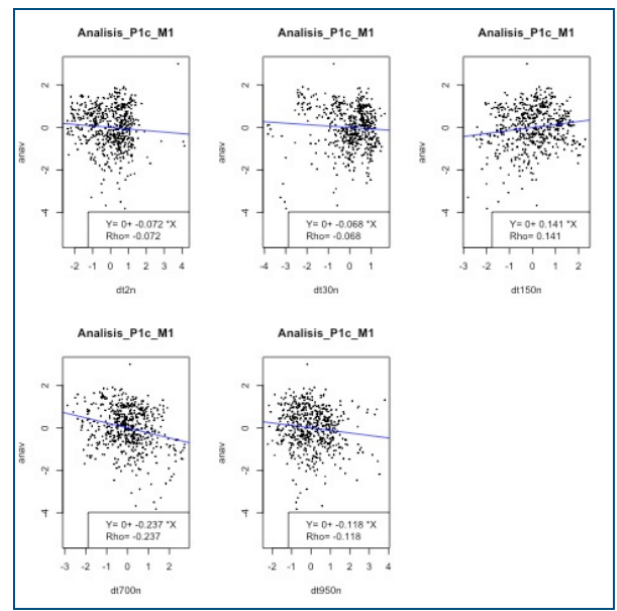

(c) Period 1c correlations with T

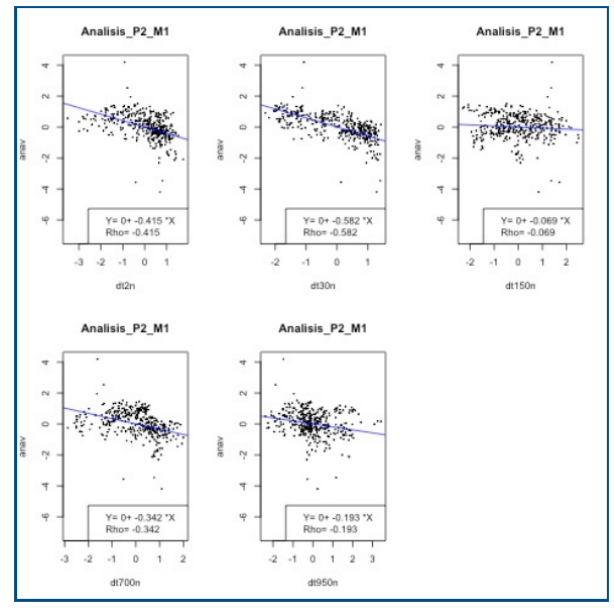

(e) Period 2 correlations with $\mathrm{T}$

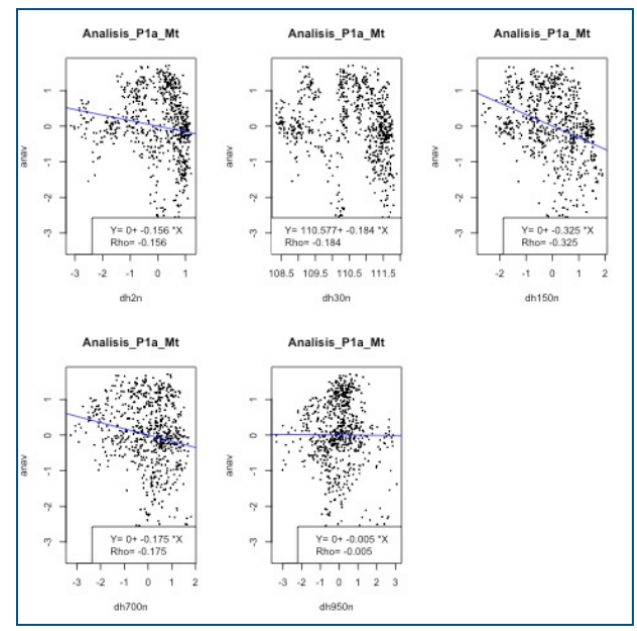

(b) Period 1a correlations with $\mathrm{H}$

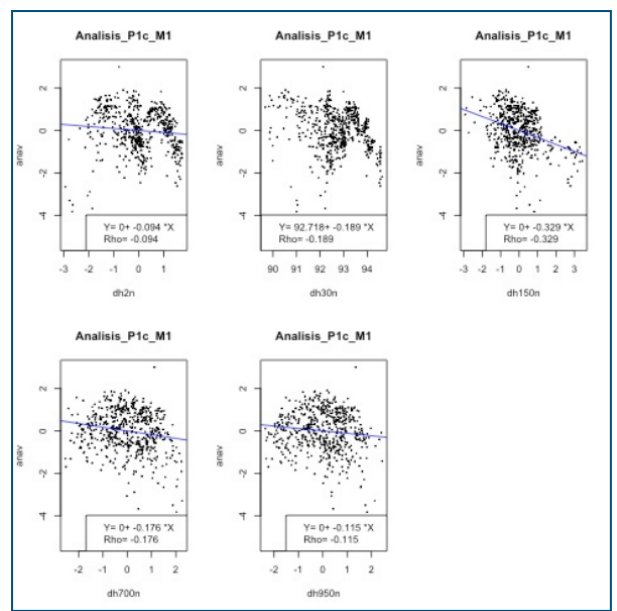

(d) Period 1c correlations with $\mathrm{H}$

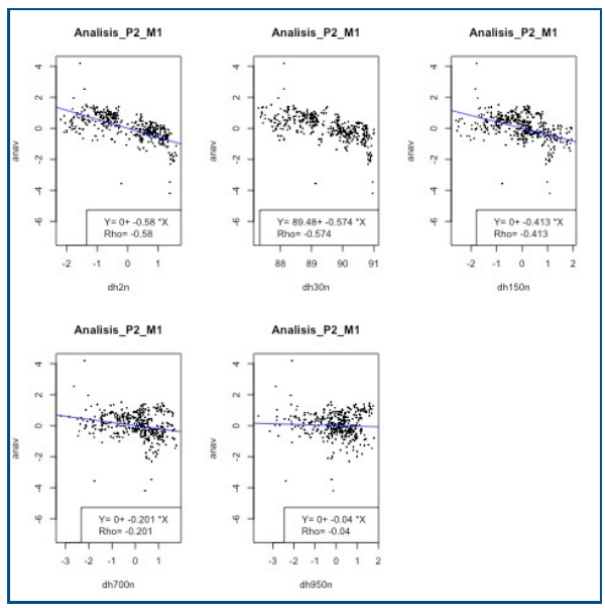

(f) Period 2 correlations with $\mathrm{H}$

Figure 5: Correlations between variations of M1 and temperature/height for the different periods. 
[3] L.I. Dorman, Cosmic rays. Variations and space explorations, North-Holland, Amsterdam, 1974.

[4] S. Sagisaka, Atmospheric effects on cosmic-ray muon intensities at deep underground depths, Il Nuovo Cimento C 9(4), 809-828 (1986).

[5] A.N. Dmitrieva et al, Temperature effect correction for muon flux at the Earth's surface: estimation of the accuracy of different methods, Journal of Physics: Conference Series 409 (1) (2013)

[6] R.R.S. De Mendonça et al, The temperature effect in secondary cosmic rays (muons) observed at the ground: analysis of the Global MUON Detetor Network data, ApJ 830(2) 88 (2016).

[7] A. Duperier, The seasonal variations of cosmic-ray intensity and temperature of the atmosphere, Proceedings of the Royal Society of London A: Mathematical, Physical and Engineering Sciences 177 204-216 (1941)

[8] A.N. Dmitrieva et al, Corrections for temperature effect for ground-based muon hodoscopes, Astroparticle Physics 34(6) 401-411 (2011)

[9] Y. Fontela et al, MIDAS: A particle identification tool for the Tragaldabas Cosmic Ray telescope, in proceedings of ICRC, POS2019.

[10] DEE, P. Dick et al, The ERAInterim reanalysis: Configuration and performance of the data assimilation system, Quarterly Journal of the royal meteorological society, 137 (656) 553-597 (2011) 